\title{
Nonlinear breatherlike localized modes in $\mathrm{C}_{60}$ nanocrystals
}

\author{
Alexander V. Savin ${ }^{1,2}$ and Yuri S. Kivshar ${ }^{2}$ \\ ${ }^{1}$ Semenov Institute of Chemical Physics, Russian Academy of Sciences, Moscow 119991, Russia \\ ${ }^{2}$ Nonlinear Physics Center, Research School of Physics and Engineering, Australian National University, Canberra ACT 0200 , Australia
}

(Received 12 December 2011; published 21 March 2012)

\begin{abstract}
We study the dynamics of nanoclusters composed of $\mathrm{C}_{60}$ fullerene molecules. We reveal that such composite nanostructures can support long-lived strongly localized nonlinear modes, which resemble discrete breathers in simple nonlinear lattices. In these localized modes, the vibrational energy density is localized predominantly at one of the $\mathrm{C}_{60}$ molecules, and it decays rapidly away from the mode. Our numerical simulations demonstrate that, at room temperatures, the lifetime of such nonlinear localized modes may exceed tens of picoseconds. Consequently, we observe that $\mathrm{C}_{60}$ nanoclusters demonstrate anomalously slow thermal relaxation when the temperature gradient decays in accord with a power law, thus violating the Cattaneo-Vernotte law of thermal conductivity.
\end{abstract}

DOI: 10.1103/PhysRevB.85.125427

PACS number(s): 63.22.Kn, 05.45.Yv, 63.20.-e

\section{INTRODUCTION}

The fullerene era was started in 1985 with the discovery of a stable $\mathrm{C}_{60}$ cluster and its interpretation as a cage structure with a soccer ball shape. ${ }^{1}$ By now, many structural, electronic, and vibrational properties of fullerenes have been studied in detail. In spite of the rapidly growing interest in new forms of fullerenes, icosahedral $\mathrm{C}_{60}$, the "most beautiful molecule," remains at the focus of active research as a prototype fullerene system. Fullerene molecule $\mathrm{C}_{60}$ keeps a prominent position primarily for the high symmetry, which greatly facilitates theoretical computations and the interpretation of experimental data, but also because it is only fullerene that can be grown in the form of large and nearly perfect single crystals. At room temperature, the diffraction experiments ${ }^{3}$ show that the $\mathrm{C}_{60}$ molecules are centered on sites of a face-centered-cubic (fcc) Bravais lattice. Each $\mathrm{C}_{60}$ molecule in the solid-state phase is coupled to other $\mathrm{C}_{60}$ molecules through weak Van der Waals forces.

By now, the internal oscillations of the fullerene molecule $\mathrm{C}_{60}$ are well studied for the case of an isolated molecule and also for $\mathrm{C}_{60}$ crystalline structures. Various theoretical and numerical methods have been developed and employed. However, the dynamics of fullerenes in the nonlinear regime and a role of nonlinear localized modes in such structures remain largely unexplored.

The main aim of this paper is to study strongly localized nonlinear oscillatory modes in such nanocrystals, which resemble the discrete breathers of much simpler nonlinear lattices. High symmetry of $\mathrm{C}_{60}$ molecules allows the existence of internal nonlinear oscillations, which do not alter substantially the spherical shape of the fullerene molecule. Such oscillations are excited by rotational degrees of freedom of pentagons and hexagons of the structure, with a variation of valent bonds. Resonant coupling of such high-amplitude oscillations with the neighboring $\mathrm{C}_{60}$ molecules will remain weak because, due to conservation of a spherical shape, the molecules will interact as large particles through Lennard-Jones potentials. This observation suggests that such structures can provide an ideal environment for the existence of nonlinear modes strongly localized in the space. ${ }^{4,5}$
Here, we demonstrate that the $\mathrm{C}_{60}$ crystals can support long-lived strongly localized nonlinear modes, which closely resemble the so-called discrete breathers in simple nonlinear lattices, and affect dramatically the heat relaxation of composite fullerene structures that obeys a power law instead of an exponential law.

The paper is organized as follows. In Sec. II, we introduce our model and the effective potentials employed in the modeling of the fullerene dynamics. Section III summarizes our results of vibrational modes of an isolated $\mathrm{C}_{60}$ molecule. While the corresponding modes were discussed previously, this is an important step of our study because the mode frequencies depend on the specific intermolecular potentials employed in the model. Sections IV and V present our original results. In particular, in Sec. IV, we demonstrate that a crystal of $\mathrm{C}_{60}$ molecules may support large-amplitude spatially localized nonlinear modes when the energy is predominantly localized on one molecule. In addition, in Sec. V we reveal the important role played by such modes in thermal relaxation of a $\mathrm{C}_{60}$ nanocrystal when the temperature gradient decays in accord to a power law, thus violating the Cattaneo-Vernotte law of thermal conductivity. Finally, Sec. VI concludes the paper.

\section{MODEL}

To analyze the dynamics of localized nonlinear modes in three-dimensional $\mathrm{C}_{60}$ crystals, we consider a cubic nanocluster of $\mathrm{C}_{60}$ molecules, as shown in Fig. 1(a). Inside of each $\mathrm{C}_{60}$ molecule of the nanocluster, two neighboring carbon atoms create a valent bond [see Fig. 2(a)]. We describe the energy associated with deformation of the valent bond created by two carbon atoms by the following interaction potential:

$$
U_{1}(\rho)=\epsilon_{1}\left\{\exp \left[-\alpha_{0}\left(\rho-\rho_{0}\right)\right]-1\right\}^{2},
$$

where $\rho$ is the length of the valent bond, $\epsilon_{1}=4.9632 \mathrm{eV}$, and $\rho_{0}=1.418 \AA$ is the energy and the equilibrium length of the bond, parameter $\alpha_{0}=1.7889 \AA^{-1}$. Each carbon atom is placed at the vertex of three planar valent angles. The corresponding deformation energy of a plane valent angle created by three atoms [see Fig. 2(b)] can be described by the interaction 


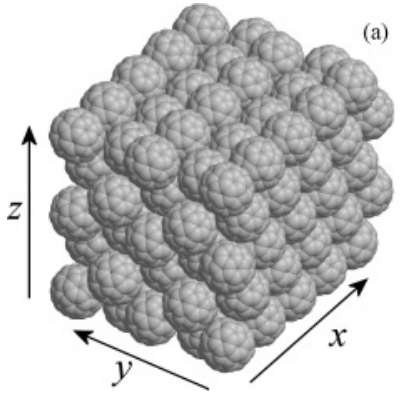

(b)

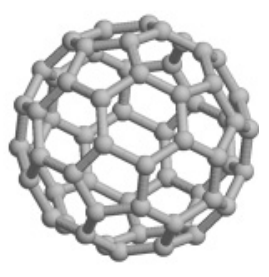

FIG. 1. (a) General view of a cubic nanocluster composed of $5 \times$ $5 \times 5$ fullerene molecules $\mathrm{C}_{60}$ (buckyballs $\mathrm{C60}-\mathrm{Ih}$ ) and (b) isolated molecule $\mathrm{C}_{60}$.

potential of the form

$$
U_{2}(\varphi)=\epsilon_{2}(\cos \varphi+1 / 2)^{2},
$$

where $\varphi$ is value of the valent angle (equilibrium value of the angle $\varphi_{0}=2 \pi / 3$ ), energy $\epsilon_{2}=1.3143 \mathrm{eV}$. Each valent bond simultaneously belongs to two planes, so that the deformation energy of the angle formed by two such planes can be described by the following interaction potential:

$$
U_{i}(\phi)=\epsilon_{i}(1-\cos \phi),
$$

where $\phi$ is the corresponding angle (in equilibrium, $\phi=0$ ), and the index $i=3,4,5$ describes the type of the dihedral angle [see Figs. 2(c), 2(d), and 2(e)]. Energy $\epsilon_{3}=\epsilon_{4}=0.499 \mathrm{eV}$, $\epsilon_{5} \ll \epsilon_{4}$, so that the latter contribution to the total energy can be neglected.

More detailed discussion and motivation of our choice of the interaction potentials $U_{1}, \ldots, U_{4}$ can be found in Ref. 6 . Such potentials have been employed for modeling of thermal conductivity of carbon nanotubes ${ }^{7,8}$ graphene nanoribbons ${ }^{6,9}$ and also in the analysis of their oscillatory modes. ${ }^{10-12}$

We describe the interaction of carbon atoms belonging to different $\mathrm{C}_{60}$ molecules by the Lennard-Jones $12-6$ potential

$$
U_{\mathrm{LJ}}(r)=4 \epsilon_{\mathrm{LJ}}\left[\left(\frac{\sigma}{r}\right)^{12}-\left(\frac{\sigma}{r}\right)^{6}\right],
$$

where $r$ is the distance between the atom centers, energy $\epsilon_{\mathrm{LJ}}=$ $0.004556 \mathrm{eV}$, and parameter $\sigma=3.851 \AA$.

To verify our results, we also employ the simpler model where all interacting potentials between the atoms are described instead of the set of the potentials $U_{1}, \ldots, U_{4}$ by the Brenner potential. ${ }^{13}$

\section{INTRAMOLECULAR MODES}

The high symmetry of an isolated, isotopically pure $\mathrm{C}_{60}$ molecule in its equilibrium configuration imposes very strong constraints on the form of the normal mode displacement patterns. All 60 sites are symmetry equivalent, and the point group is the full icosahedral group $I_{h}$. Although there are 180 degrees of freedom $(3 \times 60)$ for each $\mathrm{C}_{60}$ molecule, the icosahedral symmetry gives rise to a number of degenerate modes, so that only 46 distinct mode frequencies are expected for the $\mathrm{C}_{60}$ molecule. All vibrational modes of $\mathrm{C}_{60}$ are distributed ${ }^{14,15}$ as $2 \mathrm{~A}_{g}+\mathrm{A}_{u}+4 \mathrm{~T}_{1 g}+5 \mathrm{~T}_{1 u}+4 \mathrm{~T}_{3 g}+5 \mathrm{~T}_{3 u}+6 \mathrm{G}_{g}+6 \mathrm{G}_{u}+$ $8 \mathrm{H}_{g}+7 \mathrm{H}_{u}$.

The dynamics of an isolated $\mathrm{C}_{60}$ fullerene molecule is described by a system of $3 \times 60$ nonlinear equations of the standard form

$$
M \ddot{\mathbf{u}}=-\frac{\partial H}{\partial \mathbf{u}},
$$

where $M=12 \times 1.6603 \times 10^{-27} \mathrm{~kg}$ mass of the carbon atom, system Hamiltonian is

$$
H=\frac{1}{2} M(\dot{\mathbf{u}}, \dot{\mathbf{u}})+E(\mathbf{u}),
$$

and 180-dimensional vector $\mathbf{u}=\left\{\left(x_{n}, y_{n}, z_{n}\right)\right\}_{n=1}^{60}$ defines the coordinates of all atoms of the complex molecule. Potential energy of the molecule $E(\mathbf{u})$ is composed by all types of interactions described by the potentials $U_{1}, \ldots, U_{4}$ discussed above.

To find the equilibrium stationary state of the $\mathrm{C}_{60}$ molecule, we need to solve numerically the minimization problem for the energy functional

$$
E(\mathbf{u}) \longrightarrow \min .
$$

In the model under consideration, the molecule $\mathrm{C}_{60}$ is described by the set of coordinates $\mathbf{u}_{0}$, and it has a shape of perfect icosahedral [see Fig. 1(b)] with the radius $R=3.514 \AA$, for which all valent bonds have the equilibrium length $\rho=\rho_{0}$.

Linearizing the system equation of motion (5) near the equilibrium stationary state $\mathbf{u}=\mathbf{u}_{0}$, we obtain a system of linear equations

$$
M \ddot{\mathbf{u}}=\mathbf{A u}, \mathbf{A}=\left\{\left.\frac{\partial E}{\partial u_{i} \partial u_{j}}\right|_{\mathbf{u}=\mathbf{u}_{0}}\right\}_{i=1, j=1}^{180,180},
$$

where $\mathbf{A}$ is the $180 \times 180$ dimensional symmetrical matrix. By diagonalizing the matrix $\mathbf{A}$, we find all 180 eigenmodes of the molecule. The first six zero-frequency modes correspond to rigid translations and rotations of the molecule. Other 174 modes are generated and, in view of their degeneracy, there exist 46 distinct modes (see Table I).

The frequencies of all oscillatory eigenmodes of an isolated $\mathrm{C}_{60}$ molecule are summarized in Table I. All frequencies are located in the interval $162.1 \leqslant \omega \leqslant 1579.3 \mathrm{~cm}^{-1}$. Definitely, the model with only six parameters is not able to predict (a)

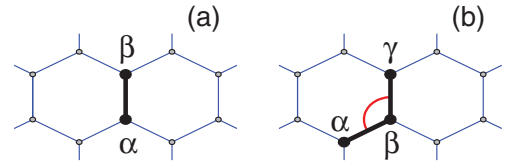

(b)

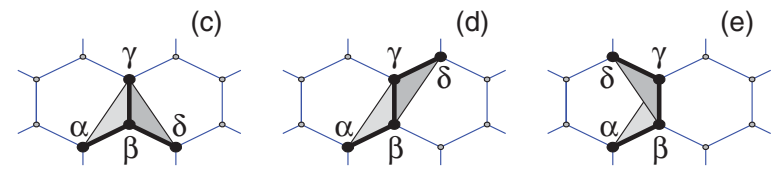

FIG. 2. (Color online) Configurations containing up to $i$ th nearest-neighbor interactions for (a) $i=1$, (b) $i=2$, (c) $i=3$, (d) $i=4$, and (e) $i=5$. 
TABLE I. Values $\omega$ (in $\mathrm{cm}^{-1}$ ) for the 46 distinct normal mode frequencies of an isolated $\mathrm{C}_{60}$ molecule ( $n$ is mode degeneracy). Notations of the modes are from Ref. 14.

\begin{tabular}{|c|c|c|c|c|c|c|c|c|c|c|c|}
\hline Mode & $\omega$ & $n$ & Mode & $\omega$ & $n$ & Mode & $\omega$ & $n$ & Mode & $\omega$ & $n$ \\
\hline $\mathrm{H}_{g}(1)$ & 162.1 & 5 & $\mathrm{H}_{g}(3)$ & 497.4 & 5 & $\mathrm{~A}_{u}$ & 776.0 & 1 & $\mathrm{G}_{g}(5)$ & 1437.5 & 4 \\
\hline $\mathrm{T}_{3 u}(1)$ & 223.2 & 3 & $\mathrm{~T}_{3 u}(2)$ & 497.8 & 3 & $\mathrm{G}_{u}(4)$ & 847.7 & 4 & $\mathrm{~T}_{1 g}(3)$ & 1454.9 & 3 \\
\hline $\mathrm{G}_{u}(1)$ & 225.5 & 4 & $\mathrm{H}_{u}(4)$ & 501.8 & 5 & $\mathrm{~T}_{3 u}(3)$ & 890.2 & 3 & $\mathrm{~A}_{g}(2)$ & 1503.2 & 1 \\
\hline $\mathrm{H}_{u}(1)$ & 278.2 & 5 & $\mathrm{~T}_{1 u}(2)$ & 503.6 & 3 & $\mathrm{H}_{g}(5)$ & 1020.0 & 5 & $\mathrm{~T}_{3 u}(5)$ & 1511.7 & 3 \\
\hline $\mathrm{H}_{g}(2)$ & 282.8 & 5 & $\mathrm{G}_{g}(3)$ & 534.7 & 4 & $\mathrm{G}_{g}(4)$ & 1039.8 & 4 & $\mathrm{~T}_{1 u}(4)$ & 1512.9 & 3 \\
\hline $\mathrm{G}_{g}(1)$ & 323.1 & 4 & $\mathrm{G}_{u}(2)$ & 535.0 & 4 & $\mathrm{H}_{u}(5)$ & 1121.8 & 5 & $\mathrm{H}_{g}(7)$ & 1518.2 & 5 \\
\hline $\mathrm{H}_{u}(2)$ & 358.6 & 5 & $\mathrm{~T}_{3 g}(2)$ & 542.5 & 3 & $\mathrm{~T}_{1 u}(3)$ & 1181.2 & 3 & $\mathrm{G}_{u}(6)$ & 1540.6 & 4 \\
\hline $\mathrm{T}_{1 u}(1)$ & 364.3 & 3 & $\mathrm{H}_{u}(3)$ & 557.1 & 5 & $\mathrm{~T}_{3 g}(4)$ & 1267.6 & 3 & $\mathrm{G}_{g}(6)$ & 1553.8 & 4 \\
\hline $\mathrm{T}_{1 g}(1)$ & 398.5 & 3 & $\mathrm{G}_{u}(3)$ & 579.3 & 4 & $\mathrm{H}_{g}(6)$ & 1270.3 & 5 & $\mathrm{H}_{u}(7)$ & 1575.0 & 5 \\
\hline $\mathrm{G}_{g}(2)$ & 413.1 & 4 & $\mathrm{~T}_{3 g}(3)$ & 603.1 & 3 & $\mathrm{~T}_{3 u}(4)$ & 1272.0 & 3 & $\mathrm{H}_{g}(8)$ & 1579.3 & 5 \\
\hline $\mathrm{A}_{g}(1)$ & 418.6 & 1 & $\mathrm{H}_{g}(4)$ & 673.7 & 5 & $\mathrm{H}_{u}(6)$ & 1376.2 & 5 & & & \\
\hline $\mathrm{T}_{3 g}(1)$ & 442.3 & 3 & $\mathrm{~T}_{1 g}(2)$ & 675.7 & 3 & $\mathrm{G}_{u}(5)$ & 1399.8 & 4 & & & \\
\hline
\end{tabular}

exactly the frequencies obtained in the framework of the firstprinciples calculations or experimental results (see Ref. 14). Nevertheless, this model demonstrates a correct structure of the eigenmode frequencies and predicts rather well the frequency band measured experimentally, $272 \leqslant \omega \leqslant 1575 \mathrm{~cm}^{-1}$.

It is important to mention that the use of the Brenner potentials ${ }^{13}$ instead of the set of the potentials $U_{1}, \ldots, U_{4}$ leads to much broader frequency band, $182.9 \leqslant \omega \leqslant 1738.9 \mathrm{~cm}^{-1}$. In addition, the Brenner potential does not predict correctly the degeneracy of the frequency spectrum, so that the equilibrium shape of the $\mathrm{C}_{60}$ molecule slightly deviates from the icosahedra shape.

For molecular dynamics simulations of the oscillatory dynamics of the $\mathrm{C}_{60}$ molecule, in the following we always employ the anharmonic models (1)-(4). The systems of the underlying equations are always nonlinear, and the results for the oscillatory eigenmodes obtained above are used only as initial conditions for a single molecule, in the simulations of strongly localized nonlinear modes of a cubic nanocrystal.

In order to model thermal oscillations of the molecule, we start from its equilibrium state and excite each of its
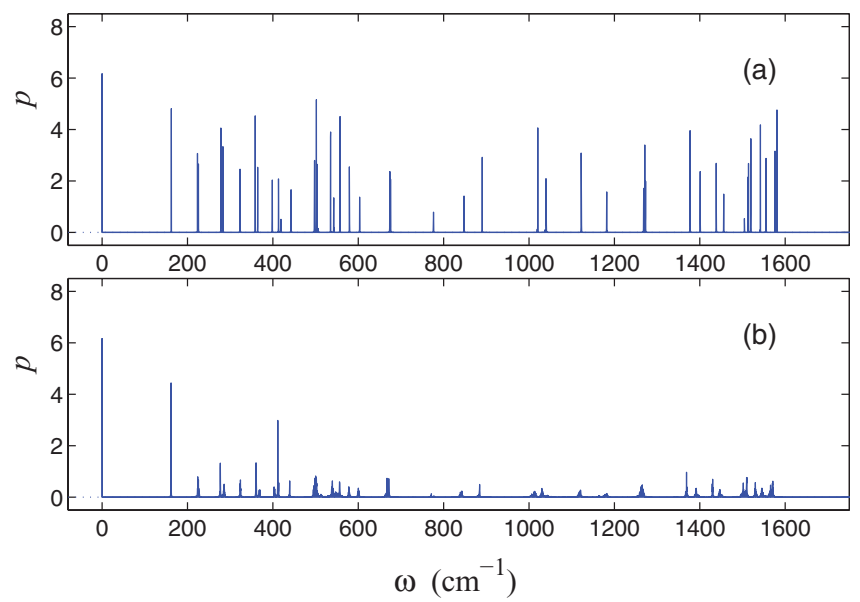

FIG. 3. (Color online) Dimensionless density of frequency spectrum of isolated molecule $\mathrm{C}_{60}$ for thermal vibrations at (a) $T=3 \mathrm{~K}$ and (b) $T=300 \mathrm{~K}$. eigenmodes with the energy $k_{B} T$. The frequency spectrum of the $\mathrm{C}_{60}$ molecule at the temperature $T=3 \mathrm{~K}$ is shown in Fig. 3(a). As expected, the spectrum is discrete, and the number of peaks coincides with the number of eigenmodes, whereas the peak positions coincide with the corresponding eigenfrequencies. At low temperatures, the oscillations are linear, so that the spectrum corresponds to a system of uncoupled linear oscillators.

If we increase the temperature to $T=30 \mathrm{~K}$, the discrete nature of the spectrum remains unchanged, but high-frequency modes demonstrate some energy exchange and interaction. For much higher temperatures, such as $T=300 \mathrm{~K}$, the discrete nature of the spectrum is lost [see Fig. 3(b)] and the oscillations become strongly nonlinear, demonstrating interaction with strong energy exchange.

\section{LOCALIZED NONLINEAR MODES}

The $\mathrm{C}_{60}$ molecules occupy fcc sites in the solid phase, and at room temperature, the rapid rotational diffusion of each molecule leads to an effective fcc crystal structure. To study localized modes of the fullerene molecule placed in a crystal, we consider a cubic nanocluster composed of $5 \times 5 \times 5$ molecules $\mathrm{C}_{60}$, as shown in Fig. 1(a).

First, we find the ground state of the nanocluster by minimizing its energy. Next, for the central molecule, we excite its $n$th eigenmode $(n=7, \ldots, 180)$ with the energy $E_{0}$ and study the decay of the kinetic energy in the cluster. Due to the nonvalent coupling between the fullerenes, the energy is transferred from one molecule to another. To model the energy spreading in an effectively infinite crystal, we introduce lossy boundary conditions, when all surface molecules experience damping with the relaxation time $t_{r}=20 \mathrm{ps}$. If the excitation of the certain eigenmode leads to the creation of a localized state, or breathinglike mode, the kinetic energy will not vanish but instead will approach a certain nonzero value. Otherwise, the initial excitation will vanish completely, so that the kinetic energy of the central molecule $E_{k} \rightarrow 0$ when $t \rightarrow \infty$.

First, we excite with the energy $E_{0}=0.2 \mathrm{eV}$ the $n$th mode $(n=7, \ldots, 180)$ of the central molecule of the fullerene crystal. Depending on the type of the modes, our numerical 

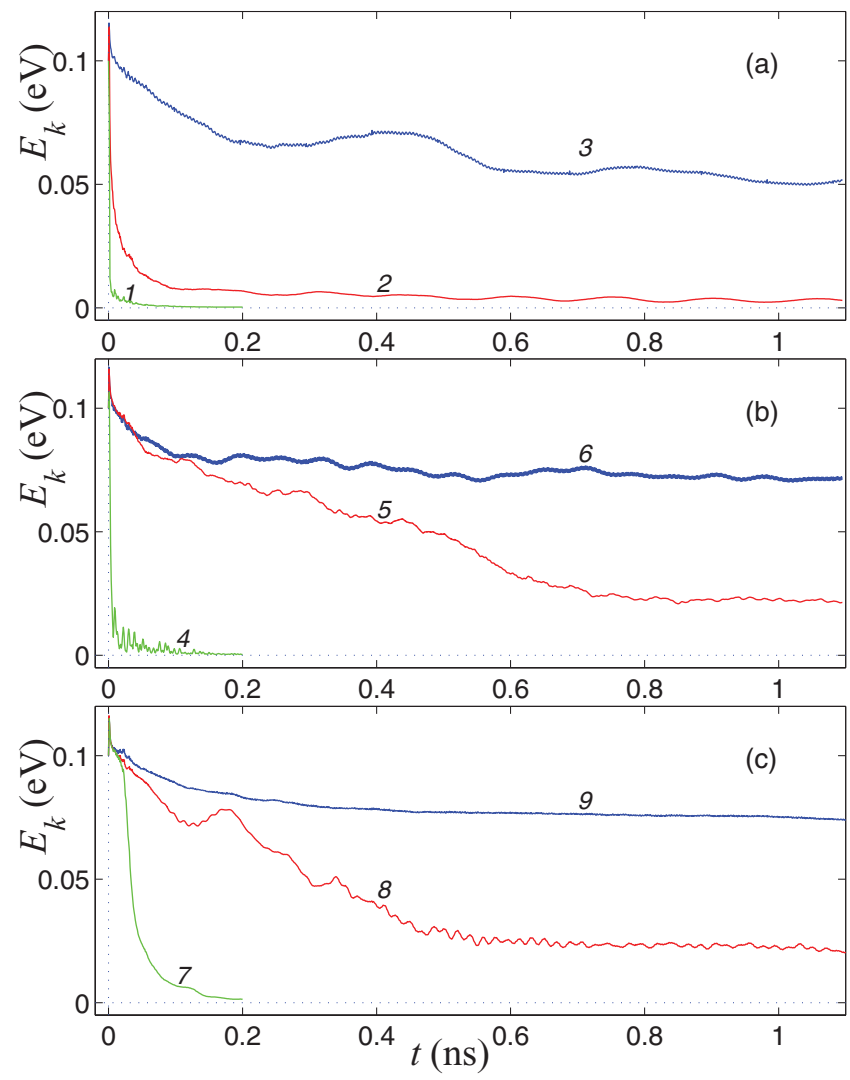

FIG. 4. (Color online) Evolution of the kinetic energy $E_{k}$ of the central fullerene $\mathrm{C}_{60}$ molecule in nanocluster composed of $5 \times 5 \times 5$ molecules packed in the form of a fcc crystal. At the initial moment $t=0$, only one eigenmode is excited for the central molecule with the energy $E_{0}=0.2 \mathrm{eV}$. (a) Curve 1 shows the decay of the mode $\mathrm{H}_{g}(1)$ (frequency $\left.\omega=162.2 \mathrm{~cm}^{-1}\right)$, curve 2 the mode $\mathrm{H}_{u}(4)(\omega=$ $\left.501.8 \mathrm{~cm}^{-1}\right)$, and curve 3 the mode $\mathrm{T}_{3 g}(1)\left(\omega=442.3 \mathrm{~cm}^{-1}\right)$; (b) curve 4 gives the dependence for the mode $\mathrm{A}_{g}(1)\left(\omega=418.6 \mathrm{~cm}^{-1}\right)$, curve 5 the mode $\mathrm{T}_{3 g}(4)\left(\omega=1267.6 \mathrm{~cm}^{-1}\right)$, curve 6 the mode $\mathrm{T}_{1 g}$ (2) $\left(\omega=675.7 \mathrm{~cm}^{-1}\right)$; (c) curve 7 describes the decay of the mode $\mathrm{A}_{u}$ (frequency $\omega=776.0 \mathrm{~cm}^{-1}$ ), curve 8 the mode $\mathrm{G}_{g}(5)$ $\left(\omega=1437.5 \mathrm{~cm}^{-1}\right)$, and curve 9 the mode $\mathrm{T}_{1 g}(3)\left(\omega=1454.9 \mathrm{~cm}^{-1}\right)$.

results reveal three different scenarios of the mode relaxation, as shown in Figs. 4(a)-4(c). The first scenario is the rapid energy relaxation and decay of the mode oscillations (see curves 1, 4, and 7); the second scenario is slow relaxation (a)
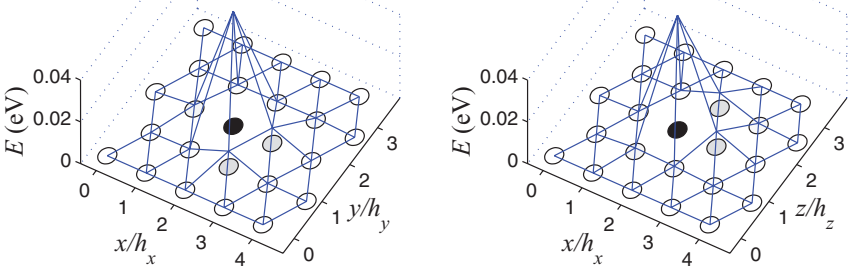

FIG. 6. (Color online) Spatial profile of the kinetic energy $E$ of the breathinglike nonlinear localized mode of the central fullerene molecule in the nanocluster of $5 \times 5 \times 5$ of $\mathrm{C}_{60}$ molecules in the planes (a) $x y$ and (b) $x z$, intersecting the central molecule (shown by a black circle). For the generation of such breathers, the eigenmode $\mathrm{T}_{3 g}(1)$ was excited with the energy $E=0.2 \mathrm{eV}$.

(curves 2, 5, and 8), and the third scenario is the generation of stationary spatially localized nonlinear modes (curves 3, 6, and 9).

The first and second scenarios are observed for the modes associated with a periodic change of the spherical shape or the size of the $\mathrm{C}_{60}$ molecule. The most interesting third scenario is observed for the modes associated with the motion of atoms along the spherical surface so that the molecule keeps its form almost unchanged. These are the eigenmodes $\mathrm{T}_{3 g}(1)$, $\mathrm{T}_{1 g}(2)$, and $\mathrm{T}_{1 g}(3)$ presented in Fig. 5. For all those modes, the molecule conserves its spherical shape and therefore the resonant interatomic interaction is reduced.

Figure 6 shows the spatial distribution of the kinetic energy of the nonlinear localized state generated by the excitation of the eigenmode $\mathrm{T}_{3 g}(1)$. The main part of the energy is concentrated at the central $\mathrm{C}_{60}$ molecule, so this state resembles discrete breathers studied earlier for simpler lattices. The nonlinear states generated by the eigenmodes $\mathrm{T}_{1 g}(2)$ and $\mathrm{T}_{1 g}(3)$ are localized even stronger. For $E>0.01$ $\mathrm{eV}$, all such modes represent strongly anharmonic oscillations with the frequency decaying with the mode amplitude. The mode $\mathrm{T}_{1 g}(2)$ carries the largest amount of energy that can reach $1 \mathrm{eV}$.

It is important to mention that the model employing the Brenner potentials predicts the existence of a larger number of localized modes. In a thermalized nanocrystal, all localized modes have a finite lifetime. Their decay occurs mainly due to the interaction with other modes of the same molecule. (a)

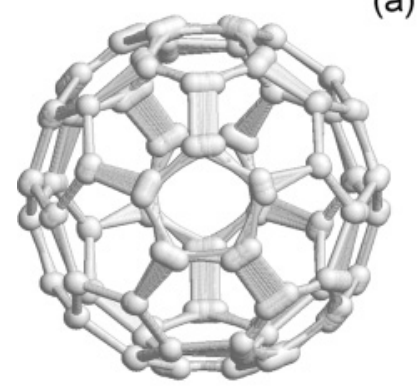

(b)

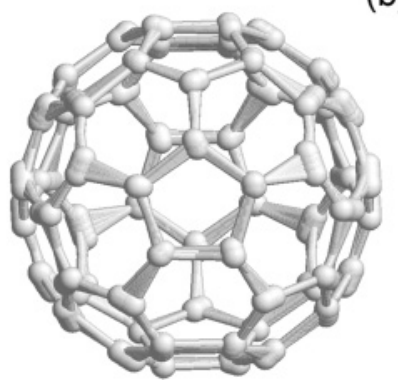

(c)

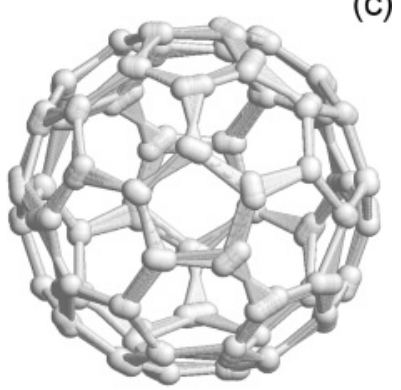

FIG. 5. Selected eigenmodes of an isolated $\mathrm{C}_{60}$ molecule, which lead to the generation of spatially localized nonlinear modes (breathers) of the fullerene crystal. (a) mode $\mathrm{T}_{3 g}(1)$ (frequency $\omega=442.3 \mathrm{~cm}^{-1}$ ); (b) mode $\mathrm{T}_{1 g}$ (2) (frequency $\omega=675.7 \mathrm{~cm}^{-1}$ ); (c) mode $\mathrm{T}_{1 g}(3)$ (frequency $\left.\omega=1454.9 \mathrm{~cm}^{-1}\right)$. 

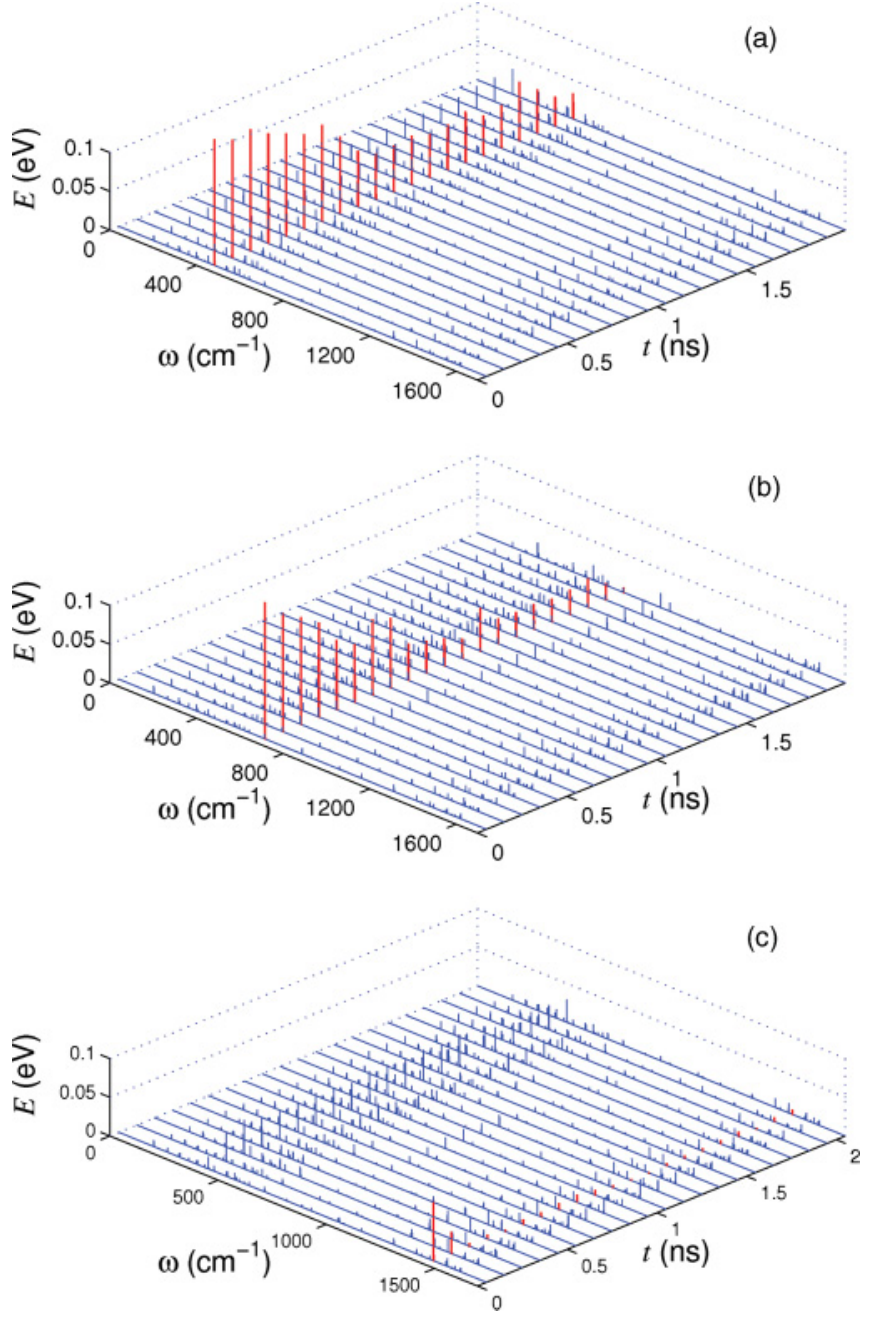

FIG. 7. (Color online) Interaction of a single large-amplitude mode (a) $\mathrm{T}_{3 g}$ (1), (b) $\mathrm{T}_{1 g}$ (2), and (c) $\mathrm{T}_{1 g}$ (3) (frequencies $\omega=$ $442.3,675.7$, and $1454.9 \mathrm{~cm}^{-1}$ ) with all other weakly thermalized eigenmodes of $\mathrm{C}_{60}$ molecules. As the initial condition at $t=0$, we excite these eigenmodes with the energy $E_{1}=0.2 \mathrm{eV}$, and all other eigenmodes with the energy $E_{0}=0.002 \mathrm{eV}$. Shown is the evolution of the energy distribution over the frequencies. The peak corresponds to a large-amplitude mode (shown in red).

Therefore, in order to define the lifetime of a nonlinear mode, it would be sufficient to study the dynamics of an isolated $\mathrm{C}_{60}$ molecule with one strongly excited mode corresponding to the nonlinear localized state and all other modes weakly thermalized.

In Fig. 7, we show the interaction of large-amplitude eigenmodes $\mathrm{T}_{3 g}(1), \mathrm{T}_{1 g}(2)$, and $\mathrm{T}_{1 g}(3)$ with all other weakly thermalized eigenmodes of the fullerene $\mathrm{C}_{60}$ molecule. As follows from those results, the energy of the localized mode decays monotonically, exciting other modes. When the initial energy of the $\mathrm{T}_{3 g}(1)$ mode is $E_{1}=0.2 \mathrm{eV}$ and the energy of all other modes is $E_{0}=0.002 \mathrm{eV}$, the localized mode loses a half of its energy for $t_{0}=1.4 \mathrm{~ns}$; for $E_{0}=0.004 \mathrm{eV}$, this time is $t_{0}=0.8 \mathrm{~ns}$; for $E_{0}=0.008 \mathrm{eV}, t_{0}=0.4 \mathrm{~ns}$; and for $E_{0}=0.016 \mathrm{eV}, t_{0}=0.1 \mathrm{~ns}$. Highly excited mode $\mathrm{T}_{1 g}(2)$ with the energy $E_{1}=0.2 \mathrm{eV}$ loses a half of its energy for $t_{0}=0.8 \mathrm{~ns}$ if energy $E_{0}=0.002 \mathrm{eV}$; for $t_{0}=0.5 \mathrm{~ns}$ if $E_{0}=0.004 \mathrm{eV}$; for $t_{0}=0.3 \mathrm{~ns}$ if $E_{0}=0.008 \mathrm{eV}$; and for $t_{0}=0.1 \mathrm{~ns}$ if $E_{0}=0.016$ $\mathrm{eV}$. The smallest lifetime is shown by the eigenmode $\mathrm{T}_{1 g}(3)$, which for the input energy $E_{1}=0.2 \mathrm{eV}$ and $E_{0}=0.002$ loses a half of its energy for $t_{0}=0.1 \mathrm{~ns}$.

Therefore, our numerical results demonstrate that in a crystal formed by $\mathrm{C}_{60}$ molecules, the spatially localized nonlinear modes are long-lived excitations, and at room temperature their lifetime is of the order of several picoseconds. We notice that some other nanostructures may also support strongly localized nonlinear modes similar to those described above. For example, these might be the modes resembling the oscillations of the fullerene $\mathrm{C}_{70}$, the oscillations at the edges of shot nanotubes, etc. However, the most symmetric structure based on the $\mathrm{C}_{60}$ molecules is probably the best candidate to search for such localized modes experimentally.

\section{THERMAL RELAXATION OF $\mathrm{C}_{60}$ CRYSTAL}

The existence of long-lived nonlinear localized collective modes in the nanocluster of $\mathrm{C}_{60}$ fullerene molecules should affect substantially their physical properties. If the thermal energy can be concentrated for long time in some localized spots, such a crystal is expected to demonstrate slow thermal relaxation. To confirm this idea, we consider thermal relaxation of a cubic nanocluster composed of $10 \times 12 \times 12 \mathrm{C}_{60}$ molecules, as shown in Fig. 8.

The 180-dimensional vector $\mathbf{u}_{n m k}$ defines the coordinates of the atoms of $(n, m, k)$ th molecule of the nanocluster $(n=$ $1, \ldots, 12 ; m=1, \ldots, 10 ; k=1, \ldots, 12)$. For thermalizing the nanocluster, we solve the system of Langevin equations

$$
M \ddot{\mathbf{u}}_{n m k}=-\frac{\partial H}{\partial u_{n m k}}-\Gamma M \dot{\mathbf{u}}_{n m k}+\Xi_{n m k},
$$

where $\Gamma=1 / t_{r}$ is the Langevin collision frequency with $t_{r}=$ $0.1 \mathrm{ps}$ being the corresponding particle relaxation time, and $\Xi_{n m k}=\left\{\xi_{n m k, i}\right\}_{i=1}^{180}$ is a 180 -dimensional vector of Gaussian distributed stochastic forces describing the interaction of atom with the thermostat.

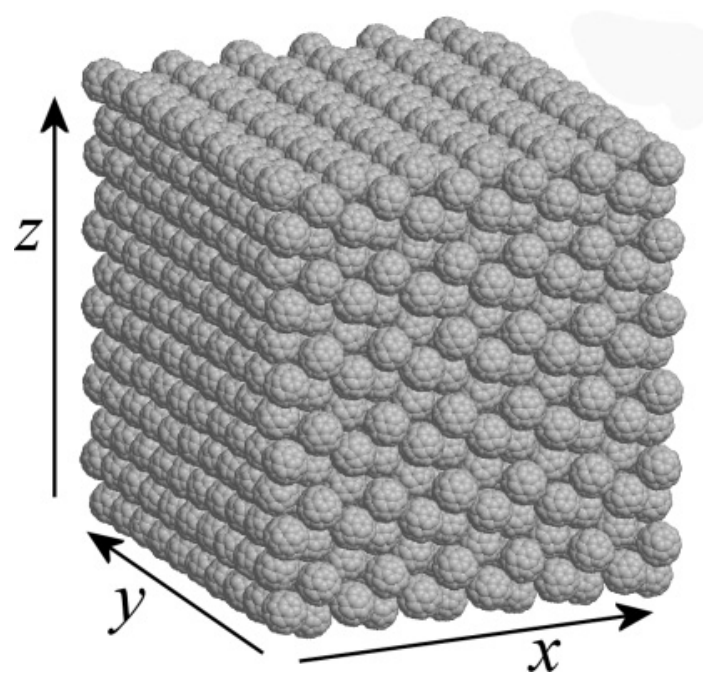

FIG. 8. Structure of nanocube created by $12 \times 12 \times 10$ fullerenes $\mathrm{C}_{60}$ molecules (with the lattice spacings $d_{x}=0.853 \mathrm{~nm}, d_{y}=$ $0.905 \mathrm{~nm}$, and $d_{z}=1.045 \mathrm{~nm}$ ). 
To analyze the thermal relaxation in the framework of classical mechanics, we introduce a random white noise with the correlation functions

$$
\left\langle\xi_{\alpha, j}\left(t_{1}\right) \xi_{\beta, l}\left(t_{2}\right)\right\rangle=2 M \Gamma k_{B} T_{\alpha} \delta_{\alpha \beta} \delta_{j l} \delta\left(t_{2}-t_{1}\right),
$$

where $T_{\alpha}$ is the temperature of the molecule with the site $\alpha=$ $(n, m, k)$. However, in the framework of semiclassical analysis, we should replace the random forces by a color noise with the correlation function satisfying the fluctuation-dissipative theorem of the quantum mechanics ${ }^{18}$

$$
I_{\xi_{\alpha, j} \xi_{\beta, l}}(\omega)=2 M \Gamma \delta_{\alpha \beta} \delta_{j l} \Theta\left(\omega, T_{\alpha}\right),
$$

where

$$
\Theta(\omega, T)=\frac{1}{2} \hbar \omega+\hbar \omega\left[\exp \left(\hbar \omega / k_{B} T\right)-1\right]^{-1} .
$$

In accord with the Wiener-Khinchin theorem, we can write

$$
\begin{aligned}
& \left\langle\xi_{\alpha, j}(t) \xi_{\beta, l}(t+\tau)\right\rangle \\
& \quad=\int_{-\infty}^{+\infty} I_{\xi_{\alpha, j} \xi_{\beta, l}}(\omega) \exp (-i \omega \tau) \frac{d \omega}{2 \pi} \\
& \quad=2 M \Gamma k_{B} T \delta_{\alpha \beta} \delta_{j l} \int_{-\infty}^{+\infty} p\left(\omega, T_{\alpha}\right) \exp (-i \omega \tau) \frac{d \omega}{2 \pi}
\end{aligned}
$$

with the dimensionless spectral density

$$
p(\omega, T)=\frac{1}{2} \frac{\hbar \omega}{k_{B} T}+\frac{\hbar \omega / k_{B} T}{\exp \left(\hbar \omega / k_{B} T\right)-1} .
$$

If $\omega_{m}$ is the maximum frequency of the vibrational eigenmodes of a molecular system, then for the temperatures $T \gg T_{m}=\hbar \omega_{m} / k_{B}$ and the frequencies $\omega \leqslant \omega_{m}$ the spectral density should satisfy the relation $p(\omega, T)=1$, namely, random forces will be acting on a molecular system as a white noise with the correlation function (7), i.e., can be described by classical fluctuations.

A simple analysis shows that for our case of the $\mathrm{C}_{60}$ fullerene crystal, the maximum frequency is $\omega_{m}=$ $1579.3 \mathrm{~cm}^{-1}$, so that the classical approach remains valid rigorously only under the unrealistic condition $T>T_{m}=$ $2272 \mathrm{~K}$. Therefore, for the temperatures $T \leqslant 500 \mathrm{~K}$, in order to obtain correct results for the Langevin equation (6), we should replace the standard classical approach by the quasiclassical analysis based on the use of the color noise with the frequency density spectrum (11). Because zero oscillations do not contribute into the thermal relaxation, we omit the first term in Eq. (11). Then, in order to obtain the initial thermalized state of the nanocluster, we solve numerically the Langevin equations (6) with the initial conditions corresponding to the equilibrium state, and to model its interaction with the thermostat we use the color noise with the correlation function (10), with the frequency density spectrum

$$
p(\omega, T)=\frac{\hbar \omega / k_{B} T}{\exp \left(\hbar \omega / k_{B} T\right)-1} .
$$

More detailed justification of the use of color noise for modeling quasiclassical fluctuations can be found in Refs. 19 and 20.

We run the simulations for $t=10 t_{r}\left(t_{r}=0.4 \mathrm{ps}\right)$ and then switch off the coupling with the thermostat, i.e., neglecting the last two terms in Eqs. (6), in order to study the thermal relaxation of the nanocluster. We thermalize the nanocluster asymmetrically to have only one side heated to the temperature $T=500 \mathrm{~K}$, whereas keeping all other atoms at the temperature $T=300 \mathrm{~K}$. To achieve this, we solve the system of Langevin equations at $T_{n m k}=500 \mathrm{~K}$ for the atoms with the index $n=1$ [for the atoms at the left-edge layer $y z$ of the nanocluster (see Fig. 8)] but for all other atoms with the indices $n=2, \ldots, 12$ we put $T_{n m k}=300 \mathrm{~K}$. In this case, we achieve a temperature difference of $\Delta T=200 \mathrm{~K}$ between the opposite $y z$ sides of the $\mathrm{C}_{60}$ nanocube. As the next step, we study the thermal relaxation of the temperature gradient.

As a result of thermal energy exchange between the fullerene molecules, temperature difference should decay monotonically. The crystal of fullerene molecules $\mathrm{C}_{60}$ has a three-dimensional structure so it should obey the Fourier law for thermal conductivity. Because time does not enter the Fourier law, it does not allow us to determine the temporal relaxation of the thermal gradient, for which we should employ the well-known generalization known as Cattaneo-Vernotte (CV) law. ${ }^{16,17}$ In its one-dimensional version, this law can be formulated through the differential equations

$$
\left(1+\tau \frac{\partial}{\partial t}\right) \vec{q}=-\kappa \vec{\nabla} T,
$$

where $\kappa$ is the standard coefficient of the thermal conductivity and $\tau$ is the characteristic relaxation time of the system. According to Eq. (13), the temperature gradient $\Delta T$ should decay in accord with an exponential dependence.

Dependence of the temperature gradient $\Delta T$ versus $t$ is shown in Fig. 9. As follows from these dependencies, the temperature difference decays very slow, in accord with the power-law dependence, so that it does not obey the CattaneoVernotte law. When we employ instead the Brenner potentials, the temperature difference decays even slower; however, it follows a similar power-law dependence.

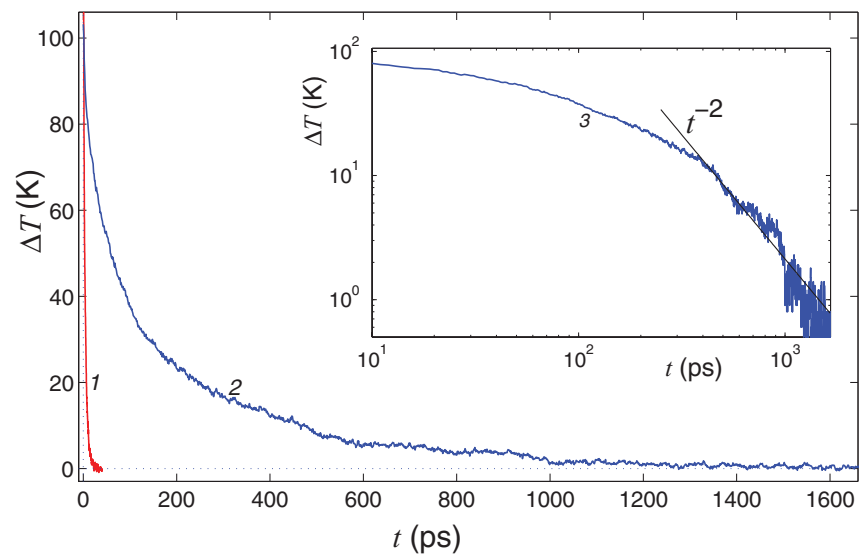

FIG. 9. (Color online) Relaxation of the thermal gradient $\Delta T$ between the opposite sides of the cubic cluster of $10 \times 12 \times 12$ fullerene $\mathrm{C}_{60}$ molecules with time $t$. Inset shows this dependence in the log-scale, with a straight line corresponding to a powerlaw dependence $\Delta T \sim t^{-2}$. Dependencies 1 correspond to the approximation of rigid molecules $\mathrm{C}_{60}$ when their internal dynamics is neglected, whereas curves 2 and 3 show the results of the full molecular dynamics simulations. 
In order to understand the reason for such an anomalous thermal relaxation as well as the role played by the internal dynamics of the molecules, we replace the $\mathrm{C}_{60}$ molecules by rigid balls and solve the same problem, assuming that the molecules in the crystal interact between each other as structureless particles. Our numerical results show (see curve 1 in Fig. 9) that in this approximation, the relaxation of the temperature difference $\Delta T$ follows an exponential dependence, in accord with the Cattaneo-Vernotte law.

For comparison, we have studied the thermal relaxation of the similar nanocrystal but composed, instead of the fullerene molecules, of identical armchair $(6,6)$ carbon nanotubes

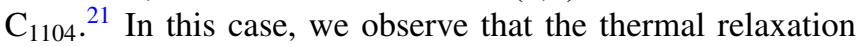
follows the familiar exponential dependence, which agrees well with the Cattaneo-Vernotte law. We notice that in the clusters of fullerene molecules $\mathrm{C}_{60}$, as well as in the clusters of $\mathrm{C}_{1104}$ carbon nanotubes but in the direction perpendicular the nanotubes, the energy transfer occurs only due to weak nonvalent interaction. Therefore, the substantial difference in the thermal relaxation of these two nanoclusters can be attributed only to the difference in the molecular structure of the molecules of fullerene and nanotubes. This difference become even larger provided we employ the Brenner potentials.

Unlike the nanocluster of $\mathrm{C}_{60}$ fullerene molecules, the long-lived breatherlike nonlinear modes do not exist in a cluster of nanotubes, so that we may attribute the slow thermal relaxation to the $\mathrm{C}_{60}$ nanoclusters to the excitation of nonlinear localized modes. The model employing the Brenner potentials predicts the existence of large number of localized modes, so that the thermal relaxation is observed to be even slower in the framework of this model.
Therefore, all these studies of limited cases and different models suggest that the anomalously slow relaxation can be attributed to the excitation of long-lived nonlinear oscillations of the $\mathrm{C}_{60}$ nanocluster.

\section{CONCLUSIONS}

We have applied the molecular-dynamics numerical simulations to study the nonlinear dynamics and thermal relaxation of crystalline structures composed of $\mathrm{C}_{60}$ fullerene molecules. We have revealed that such complex nanoclusters support a special type of strongly localized nonlinear modes resembling discrete breathers of simple nonlinear lattices. For such modes, the kinetic energy is localized primarily in the rotational modes of a single $\mathrm{C}_{60}$ molecule and it decays slowly inside the fullerene nanocrystal. Importantly, our results have demonstrated that at room temperatures, the lifetime of such nonlinear localized modes may exceed tens of picoseconds. The existence of such long-lived localized nonlinear states in the nanoclusters explains the anomalously slow thermal relaxation observed in such structures when the temperature gradient decays in accord with the power, but not exponential, law, thus violating the Cattaneo-Vernotte law of thermal conductivity.

\section{ACKNOWLEDGMENTS}

A. Savin acknowledges a warm hospitality of the Nonlinear Physics Center at the Australian National University, and he thanks the Joint Supercomputer Center of the Russian Academy of Sciences for the use of their computer facilities. The work was supported by the Australian Research Council.
${ }^{1}$ H. W. Kroto, J. Heath, S. C. O'Brien, R. F. Curl, and R. E. Smalley, Nature (London) 318, 162 (1985).

${ }^{2} \mathrm{H}$. Aldersey-Williams, The Most Beautiful Molecule: The Discovery of the Buckyball (Wiley, New York, 1995), pp. 2-3.

${ }^{3}$ R. M. Fleming, T. Siegrist, P. Marsh, B. Hessen, A. R. Kortan, D. W. Murphy, R. C. Haddon, R. Tycko, G. Dabbagh, A. M. Mujsce, M. L. Kaplan, and S. M. Zahurak, Mater. Res. Soc. Symp. Proc. 206, 691 (1991).

${ }^{4}$ D. K. Campbell, S. Flach, and Y. S. Kivshar, Phys. Today 57(1), 43 (2004).

${ }^{5}$ S. Flach and A. V. Gorbach, Phys. Rep. 467, 1 (2008).

${ }^{6}$ A. V. Savin, Y. S. Kivshar, and B. Hu, Phys. Rev. B 82, 195422 (2010).

${ }^{7}$ A. V. Savin, Y. S. Kivshar, and B. Hu, Europhys. Lett. 88, 26004 (2009).

${ }^{8}$ A. V. Savin, B. Hu, and Y. S. Kivshar, Phys. Rev. B 80, 195423 (2009).

${ }^{9}$ A. V. Savin and Y. S. Kivshar, Lett. Mater. 1, 3 (2011) [Pisma o Materialakh 1, 3 (2011)].
${ }^{10}$ A. V. Savin and Y. S. Kivshar, Appl. Phys. Lett. 94, 111903 (2009).

${ }^{11}$ A. V. Savin and Y. S. Kivshar, Europhys. Lett. 89, 46001 (2010).

${ }^{12}$ A. V. Savin and Y. S. Kivshar, Phys. Rev. B 81, 165418 (2010).

${ }^{13}$ D. W. Brenner, O. A. Shenderova, J. A. Harrison, S. J. Stuart, B. Ni, and S. B. Sinnott, J. Phys.: Condens. Matter 14, 783 (2002).

${ }^{14} \mathrm{~J}$. Menendez and J. B. Page, in Light Scattering in Solids VIII, edited by M. Cardona and G. Guntherodt (Springer, Heidelberg, 2000), pp. 27-95.

${ }^{15}$ D. E. Weeks and W. G. Harter, J. Chem. Phys. 90, 4744 (1989).

${ }^{16}$ P. Vernotte, C. R. Hebd. Seances Acad. Sci. 246, 3154 (1958).

${ }^{17}$ C. Cattaneo, C. R. Hebd. Seances Acad. Sci. 247, 431 (1958).

${ }^{18}$ H. B. Callen and T. A. Welton, Phys. Rev. 83, 34 (1951).

${ }^{19}$ S. Buyukdagli, A. V. Savin, and B. Hu, Phys. Rev. E 78, 066702 (2008).

${ }^{20}$ H. Dammak, Y. Chalopin, M. Laroche, M. Hayoun, and J.-J. Greffet, Phys. Rev. Lett. 103, 190601 (2009).

${ }^{21}$ A. V. Savin and Y. S. Kivshar, Appl. Phys. Lett. 98, 193106 (2011). 